\title{
Liraglutide reduces fatty degeneration in hepatic cells via the AMPK/SREBP1 pathway
}

\author{
YAN-GUI WANG ${ }^{1,2}$ and TIAN-LUN YANG ${ }^{1}$ \\ ${ }^{1}$ Department of Cardiology, Xiangya Hospital, Central South University, Changsha, Hunan 410078; \\ ${ }^{2}$ Department of Geriatrics, Hunan Provincial People's Hospital, Changsha, Hunan 410001, P.R. China
}

Received January 28, 2015; Accepted August 20, 2015

DOI: $10.3892 /$ etm.2015.2741

\begin{abstract}
Recent studies have suggested that liraglutide could have a potential function in improving non-alcoholic fatty liver disease (NAFLD); however, the underlying molecular mechanism remains unclear. The aim of the present study was to investigate the role of the AMP-activated protein kinase (AMPK)/sterol regulatory element binding protein 1 (SREBP1) pathway in mediating the effect of liraglutide in reducing fatty degeneration in an in vitro NAFLD model. To resemble the NAFLD condition in vitro, L-02 cells were treated with $0.5 \mathrm{mM}$ free fatty acids (FFAs) for $24 \mathrm{~h}$. Liraglutide could affect the expression of AMPK $\alpha 1$, phosphorylated AMPK $\alpha 1$ and SREBP1 in a dose-dependent manner in FFA-exposed L-02 cells, as demonstrated by western blot analysis. The intracellular lipid accumulation was significantly decreased, as shown by oil red $\mathrm{O}$ staining. A significant decrease in the content of triglyceride and total cholesterol was observed when the FFA-exposed L-02 cells were incubated with liraglutide. In addition, the increased expression of liver-type fatty acid-binding protein in FFA-exposed L-02 cells was suppressed by liraglutide. These effects were reversed by compound $\mathrm{C}$, an AMPK inhibitor. In conclusion, this study has demonstrated that liraglutide can reduce fatty degeneration induced by FFAs in hepatocytes, and this effect may be partially mediated by the AMPK/SREBP1 pathway.
\end{abstract}

\section{Introduction}

Non-alcoholic fatty liver disease (NAFLD) is a clinical syndrome characterized by the accumulation of excess fat in the liver of individuals that drink little or no alcohol. The prevalence of NAFLD is $15-30 \%$ in Western populations (1-4) and 6-25\%

Correspondence to: Professor Tian-Lun Yang, Department of Cardiology, Xiangya Hospital, Central South University, 87 Xiangya Road, Changsha, Hunan 410078, P.R. China E-mail: tlyang135@163.com

Key words: liraglutide, non-alcoholic fatty liver disease, hepatic cells, AMP-activated protein kinase/sterol regulatory element binding protein 1 pathway, fatty degeneration in Asian populations (5). NAFLD is associated with metabolic syndrome, obesity, diabetes and cardiovascular disease (6). Currently, no standard for the treatment of NAFLD exists, and treating risk factors remains the focus of managing NAFLD (7).

Liraglutide is a potent, long-acting synthetic analogue of the human glucagon-like peptide 1 (GLP-1) molecule. It shares 97\% sequence identity with human GLP-1. Liraglutide has previously been used for the treatment of diabetes (8); however, a recent study reported that liraglutide could improve oxidative stress and lipid peroxidation and decrease transforming growth factor- $\beta 1$ and tumor necrosis factor- $\alpha$ expression in rats with NAFLD (9). In a study by Olaywi et al (10), it was revealed that liraglutide induced improvements in transaminases, as well as histology, in patients with non-alcoholic steatohepatitis. These studies have suggested the potential function of liraglutide in improving NAFLD.

The AMP-activated protein kinase (AMPK) signaling pathway plays a key role in regulating hepatic lipid metabolism. It has been revealed that AMPK coordinates the long-term adaptation of lipid metabolism by downregulating the transcriptional factor sterol regulatory element binding protein 1 (SREBP1) (11,12).

To the best of our knowledge, studies on the molecular mechanisms underlying the liraglutide-induced improvement in NAFLD are limited (13). Whether liraglutide reduces fatty degeneration in hepatic cells via the AMPK /SREBP1 pathway remains unclear; therefore, a well-described model of in vitro NAFLD was established in the present study using a normal human hepatocyte-derived cell line, with the aim of investigating the role of the AMPK/SREBP1 pathway in mediating the liraglutide-induced reduction in fatty degeneration.

\section{Materials and methods}

Cell culture. The L-02 human normal liver cell line was purchased from the American Type Culture Collection (Manassas, VA, USA). The cells were cultured in Dulbecco's modified Eagle's medium (Gibco-BRL, Grand Island, NY, USA) supplemented with $10 \%$ fetal bovine serum (Gibco-BRL) at $37^{\circ} \mathrm{C}$ with $5 \% \mathrm{CO}_{2}$. Oleic acid (OA) and palmitic acid (PA) were purchased from Sigma-Aldrich (St. Louis, MO, USA) and added to the culture medium at the concentration of $0.5 \mathrm{mM}$ in a 2:1 molar ratio. Liraglutide was provided by Novo Nordisk (Copenhagen, Denmark) and diluted to concentrations of 


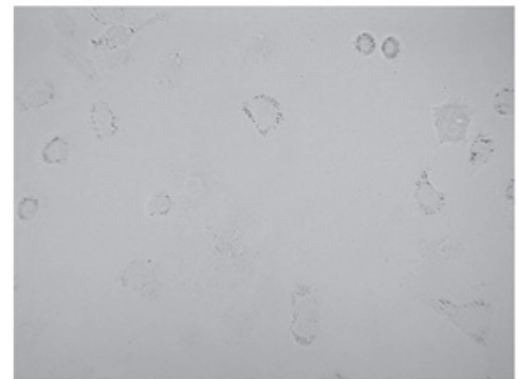

Control

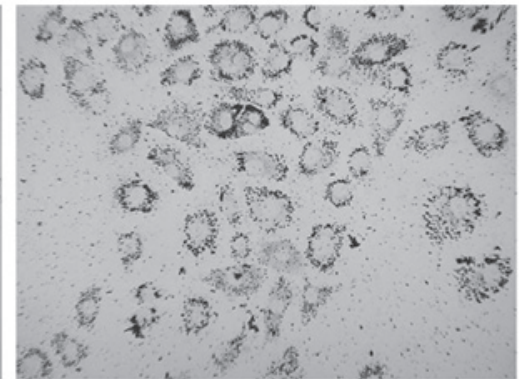

FFAs

Figure 1. Oil red O staining of L-02 cells following FFA treatment (magnification, x400). FFA, free fatty acid.

$0.1,0.5,1.0$ and $2.0 \mathrm{nM}$. Compound $\mathrm{C}$ was obtained from Calbiochem (San Diego, CA, USA) and used at a concentration of $20 \mu \mathrm{M}$. Cells were incubated with free fatty acids (FFAs), liraglutide or compound $\mathrm{C}$ alone or in combination.

Oil red $O$ staining. The cells were fixed with $10 \%$ formaldehyde for $10 \mathrm{~min}$ and then washed with phosphate-buffered saline (PBS). Oil red O solution (60\% oil red O dye and $40 \%$ water) (Sigma-Aldrich) was filtered and added to stain the cells at room temperature for $15 \mathrm{~min}$. PBS was then used to remove the unbound dye, and the cells were observed under the microscope (TE200; Nikon Corp., Tokyo, Japan).

Measurement of triglyceride $(T G)$ and total cholesterol $(T C)$. The TG quantification and cholesterol assay kits were purchased from Abcam (Cambridge, MA, USA). The levels of TG and TC were determined according to the manufacturer's instructions. Briefly, a standard curve was prepared, and the cells were lysed. For the measurement of TG, $2 \mu 1$ lipase was added to each well and incubated at room temperature for $20 \mathrm{~min}$. A total of $50 \mu \mathrm{l}$ reaction mix, including $46 \mu \mathrm{l} \mathrm{TG}$ assay buffer, $2 \mu \mathrm{l} \mathrm{TG}$ probe and $2 \mu \mathrm{l} \mathrm{TG}$ enzyme mix, was then added and incubated at room temperature for 30-60 min. For the measurement of TC, $50 \mu \mathrm{l}$ reaction mix, containing $44 \mu \mathrm{l}$ cholesterol assay buffer, $2 \mu \mathrm{l}$ cholesterol probe, $2 \mu \mathrm{l}$ enzyme mix and $2 \mu \mathrm{l}$ cholesterol esterase, was added to each well and incubated at $37^{\circ} \mathrm{C}$ for $60 \mathrm{~min}$. The optical density at $570 \mathrm{~nm}$ was measured using a microtiter plate reader (ELx800NB; BioTek Instruments, Inc., Winooski, VT, USA).

Western blot analysis. The cells were lysed in radioimmunoprecipitation assay buffer (Sangon Biotech, Shanghai, China), and protein concentrations were quantified via the bicinchoninic acid (BCA) assay method using a BCA Protein Assay kit (Beyotime Institute of Biotechnology, Shanghai, China). Proteins were separated using $12 \%$ sodium dodecyl sulfate-polyacrylamide gel and transferred onto polyvinylidene difluoride membranes (Millipore, Billerica, MA, USA). The membranes were incubated in Tris-buffered saline containing $5 \%$ non-fat milk at $37^{\circ} \mathrm{C}$ for $2 \mathrm{~h}$. The membranes were then incubated at $37^{\circ} \mathrm{C}$ for $1 \mathrm{~h}$ with the following primary antibodies: Rabbit monoclonal to AMPKa1 (1:1000; ab32047), rabbit polyclonal to AMPK $\alpha 1$ (phospho S487) (1:1,000; ab131357), rabbit monoclonal to AMPK $\alpha 1$ (phospho S496) (1:1,000; ab92701), rabbit polyclonal to SREBP1 (1:500; ab193318), rabbit polyclonal to SREBP1 (phospho S372) (1:500; ab140483), rabbit polyclonal to SREBP1 (phospho S439) (1:500; ab138663), rabbit monoclonal to liver-type fatty acid-binding protein (L-FABP) (1:1,000; ab129203) (all Abcam) and mouse monoclonal to $\beta$-actin (1:1,000; sc-47778; Santa Cruz Biotechnology, Inc., Santa Cruz, CA, USA). After washing with PBS, the membranes were incubated with the secondary antibodies [goat anti-rabbit $\mathrm{IgG} /$ horseradish peroxidase (HRP) and goat anti-mouse IgG/HRP; Santa Cruz Biotechnology, Inc.] at $37^{\circ} \mathrm{C}$ for $1 \mathrm{~h}$. The signals were detected using enhanced chemiluminescence (ECL) (ECL western blotting kit; Pierce, Rockford, IL, USA).

Statistical analysis. Data were analyzed using SPSS software, version 19.0 (IBM SPSS, Armonk, NY, USA). Data are expressed as the mean \pm standard deviation. The differences between two groups were analyzed using the Student's t-test. $\mathrm{P}<0.05$ was considered to indicate a statistically significant difference.

\section{Results}

FFA treatment induces lipid accumulation in L-02 cells. L-02 cells were treated with $0.5 \mathrm{mM}$ FFAs for $24 \mathrm{~h}$, and oil red O staining was then performed. As shown in Fig. 1, no lipid droplets were detected in the L-02 cells in the control group; however, a large quantity of red lipid droplets was observed in the L-02 cells in the FFA group. In addition, the intracellular levels of TG and TC were significantly increased by treatment with FFAs ( $\mathrm{P}<0.01$; Fig. 2).

FFA treatment induces L-FABP expression in L-02 cells. L-02 cells were treated with $0.5 \mathrm{mM}$ FFAs for $24 \mathrm{~h}$, and the expression of L-FABP in the L-02 cells was then analyzed using western blot analysis. It was found that the L-FABP expression was significantly different between the control and the FFA group. The protein expression of L-FABP was significantly upregulated following FFA treatment $(\mathrm{P}<0.01$; Fig. 3$)$.

FFA treatment affects the AMPK/SREBP1 pathway in L-O2 cells. As shown in Fig. 4, incubation of the L-02 cells with FFAs for $24 \mathrm{~h}$ resulted in the decreased expression of AMPK $\alpha 1$ and a reduction in the phosphorylation of AMPK $\alpha 1(\mathrm{P}<0.01)$. The expression of SREBP1 was increased significantly by treatment with FFAs $(\mathrm{P}<0.01)$.

Liraglutide activates the AMPK/SREBP1 pathway in FFA-exposed L-02 cells. To determine the effect of liraglutide 

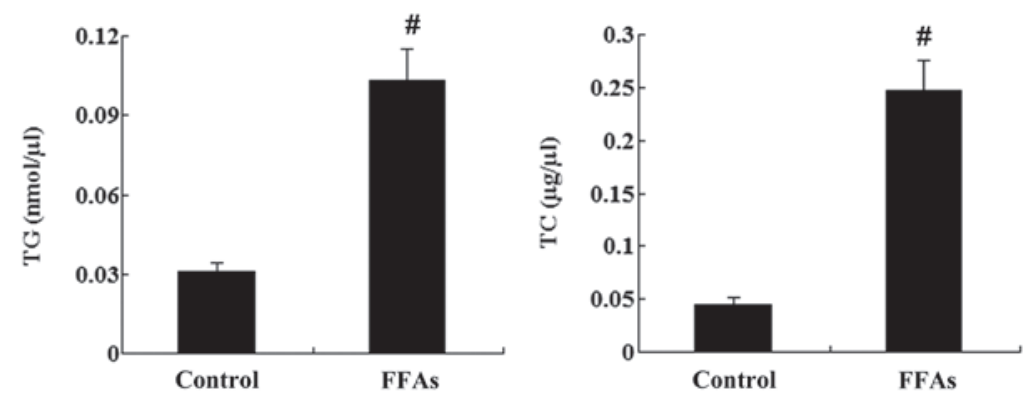

Figure 2. Intracellular levels of TG and TC in L-02 cells following FFA treatment. ${ }^{\#} \mathrm{P}<0.01$ vs. control group. TG, triglyceride; TC, total cholesterol; FFA, free fatty acid.

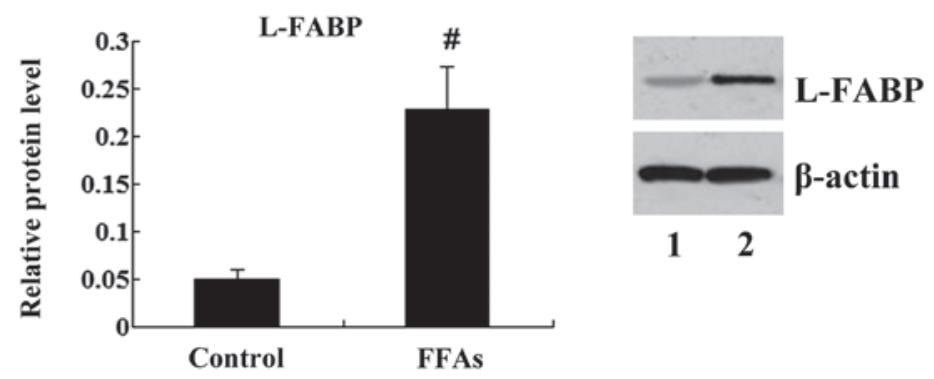

Figure 3. Expression of L-FABP protein in L-02 cells following FFA treatment. $\beta$-actin expression was used as an internal control. Lane 1, control group; lane 2, FFA group. ${ }^{*} \mathrm{P}<0.01$ vs. control group. L-FABP, liver-type fatty acid-binding protein; FFA, free fatty acid.
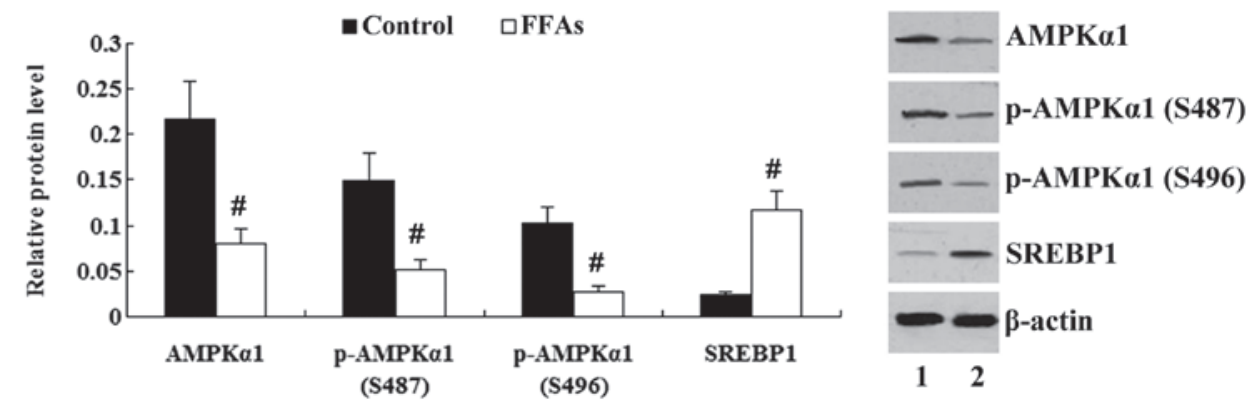

Figure 4. Expression of AMPK $\alpha 1$, p-AMPK $\alpha 1$ and SREBP1 proteins in L-02 cells following FFA treatment. $\beta$-actin expression was used as an internal control. Lane 1, control group; lane 2, FFA group. " $\mathrm{P}<0.01$ vs. control group. p-AMPK $\alpha 1$, phosphorylated AMP-activated protein kinase $\alpha 1$; SREBP1, sterol regulatory element binding protein 1 ; FFA, free fatty acid.

on the AMPK/SREBP1 pathway, FFA-exposed L-02 cells were incubated with liraglutide at different concentrations $(0.1,0.5,1.0$ and $2.0 \mathrm{nM})$. The expression of AMPK $\alpha 1$ and SREBP1 was determined using western blot analysis. As shown in Fig. 5, the expression of AMPK $\alpha 1$ and phosphorylated (p-)AMPK $\alpha 1$ (S496) was significantly increased in the FFA-exposed L-02 cells following treatment with liraglutide $(0.5-2.0 \mathrm{nM})(\mathrm{P}<0.05)$. Liraglutide at the concentrations of 1.0 and $2.0 \mathrm{nM}$ significantly upregulated $\mathrm{p}-\mathrm{AMPK} \alpha 1$ (S487) expression $(\mathrm{P}<0.01)$; however, the expression of SREBP1 in FFA-exposed L-02 cells was significantly decreased by treatment with 1.0 and $2.0 \mathrm{nM}$ liraglutide $(\mathrm{P}<0.01)$ (Fig. 5).

Liraglutide reduces lipid accumulation and L-FABP expression in FFA-exposed L-O2 cells. Next, the effect of liraglutide on lipid accumulation and L-FABP expression in FFA-exposed L-02 cells was investigated. FFA-exposed L-02 cells were incubated with liraglutide $(1.0 \mathrm{nM})$, and oil red $\mathrm{O}$ staining was then performed. As shown in Fig. 6, liraglutide decreased the number of lipid droplets in the FFA-exposed L-02 cells. The intracellular levels of TG and TC were also decreased significantly in the FFA-exposed L-02 cells following treatment with liraglutide $(1.0 \mathrm{nM})(\mathrm{P}<0.01$; Fig. 7). Western blotting results demonstrated that the incubation of FFA-exposed L-02 cells with liraglutide resulted in a decreased expression of L-FABP protein $(\mathrm{P}<0.05$; Fig. 8$)$.

AMPK/SREBP1 pathway is involved in the effect of liraglutide on lipid accumulation and L-FABP expression in FFA-exposed L-O2 cells. To investigate whether the AMPK/SREBP1 pathway was involved in the effect of liraglutide on lipid accumulation and L-FABP expression in the FFA-exposed L-02 cells, compound C, an AMPK inhibitor was added to treat the cells. As indicated in Fig. 9, AMPK $\alpha 1$ 

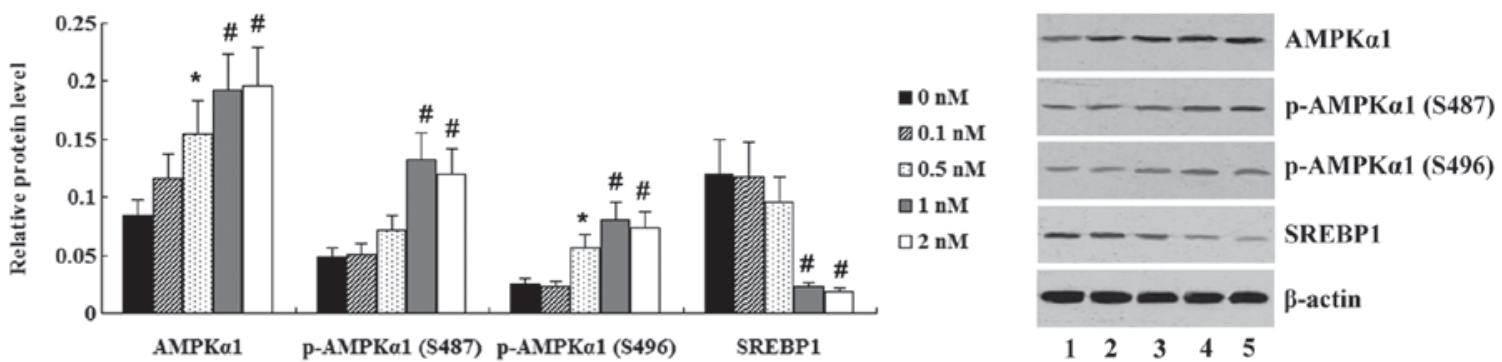

Figure 5. Expression of AMPK $\alpha 1$, p-AMPK $\alpha 1$ and SREBP1 proteins in FFA-exposed L-02 cells following treatment with different concentrations of liraglutide. $\beta$-actin expression was used as an internal control. Lanes 1-5 represent cells that were treated with $0,0.1,0.5,1.0$ and 2.0 nM liraglutide, respectively. ${ }^{*} \mathrm{P}<0.05$ and ${ }^{~} \mathrm{P}<0.01$ vs. $0 \mathrm{nM}$ group. p-AMPK $\alpha 1$, phosphorylated AMP-activated protein kinase $\alpha 1$; SREBP1, sterol regulatory element binding protein 1 ; FFA, free fatty acid.

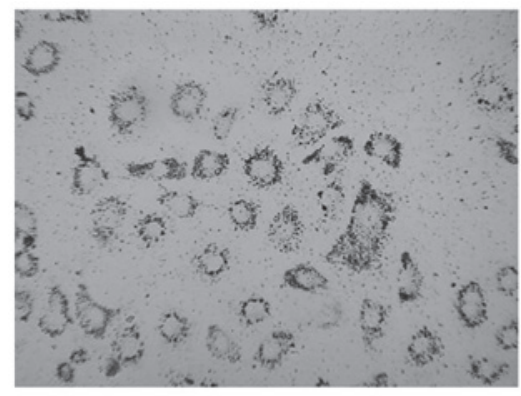

FFAs

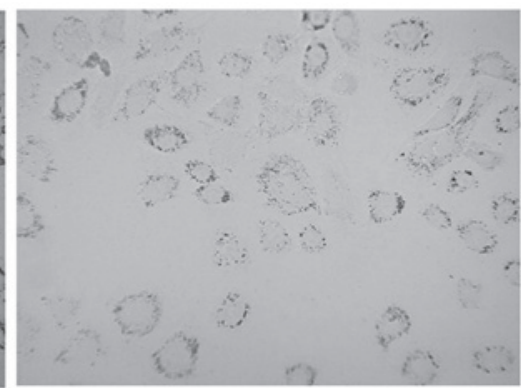

FFAs+lirag

Figure 6. Oil red O staining of FFA-exposed L-02 cells following treatment with liraglutide (magnification, x400). FFA, free fatty acid; lirag, liraglutide.
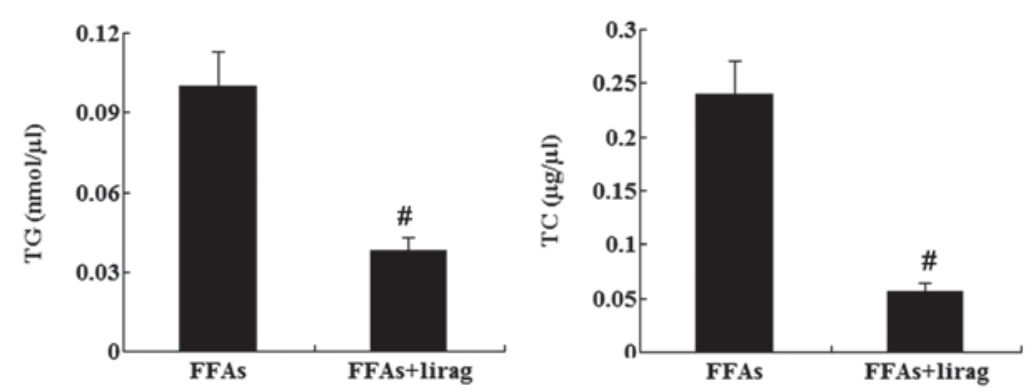

Figure 7. Intracellular levels of TG and TC in FFA-exposed L-02 cells following treatment with liraglutide. ${ }^{*} \mathrm{P}<0.01$ vs. FFA group. TG, triglyceride; TC, total cholesterol; FFA, free fatty acid; lirag, liraglutide.
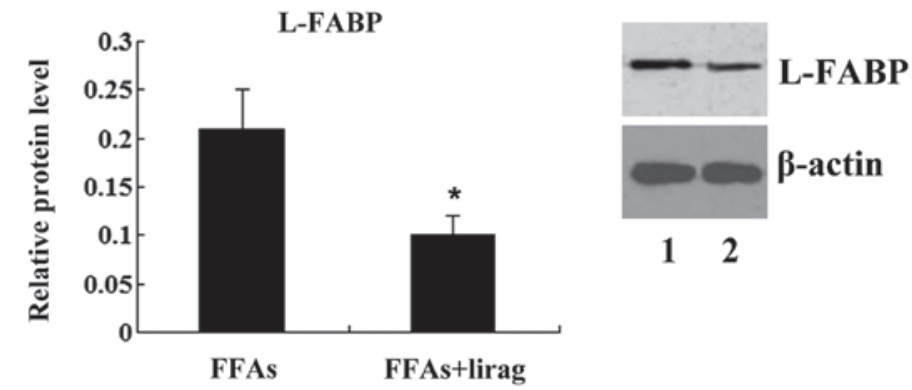

12

Figure 8. Expression of L-FABP protein in FFA-exposed L-02 cells following treatment with liraglutide. $\beta$-actin expression was used as an internal control. Lane 1, FFA group; lane 2, FFAs + lirag group. "P<0.05 vs. FFA group. L-FABP, liver-type fatty acid-binding protein; FFA, free fatty acid; lirag, liraglutide.

and $\mathrm{p}$-AMPK $\alpha 1$ protein levels were significantly decreased $(\mathrm{P}<0.05)$, while SREBP1 and $\mathrm{p}$-SREBP1 protein levels were significantly increased $(\mathrm{P}<0.01)$, in the FFA-exposed L-02 cells in the liraglutide + compound $\mathrm{C}$ group compared with those in the liraglutide group. Oil red O staining showed that liraglutide reduced the number of lipid droplets in the 

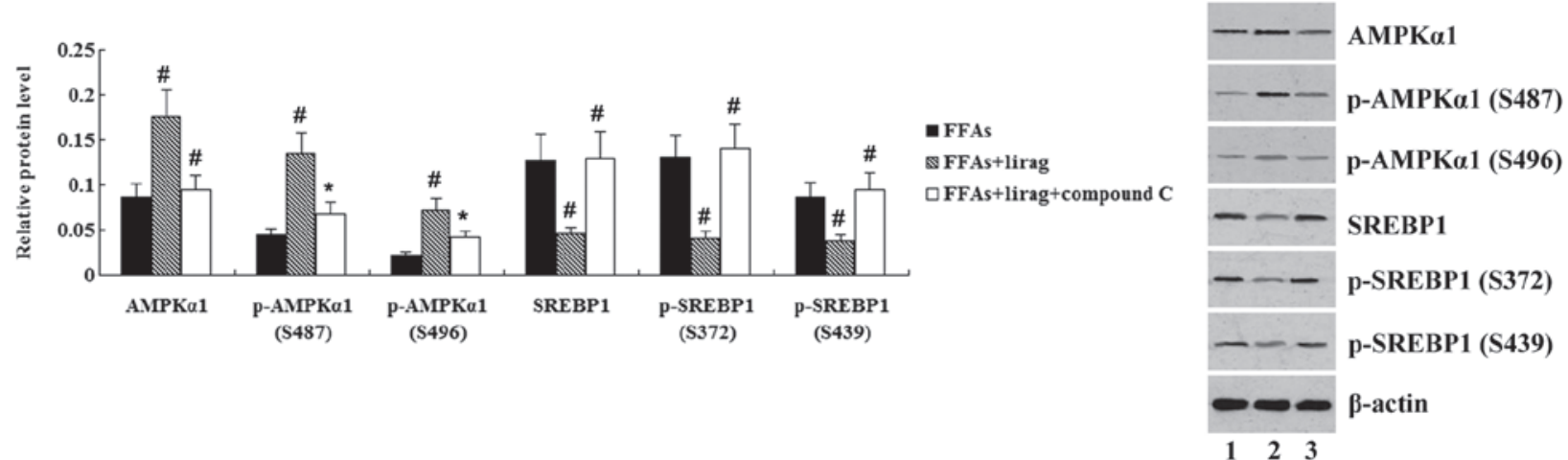

Figure 9. Expression of AMPK $\alpha 1$, p-AMPK $\alpha 1$, SREBP1 and p-SREBP1 proteins in FFA-exposed L-02 cells following treatment with liraglutide and compound C. $\beta$-actin expression was used as an internal control. Lane 1, FFA group; lane 2, FFAs + lirag group; lane 3, FFAs + lirag + compound C group. ${ }^{*} \mathrm{P}<0.05$ and ${ }^{\#} \mathrm{P}<0.01$ vs. FFA group. p-AMPK $\alpha 1$, phosphorylated AMP-activated protein kinase $\alpha 1$; SREBP1, sterol regulatory element binding protein 1; FFA, free fatty acid; lirag, liraglutide.

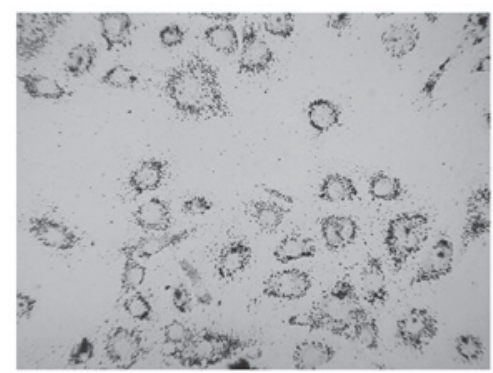

FFAs

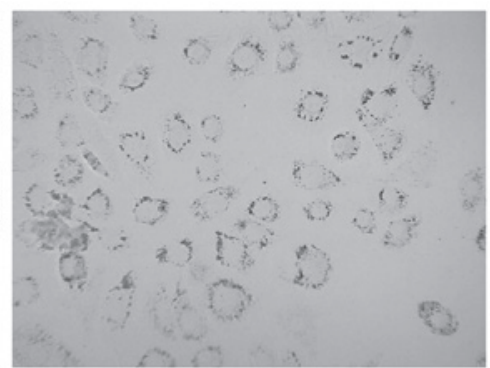

FFAs+lirag

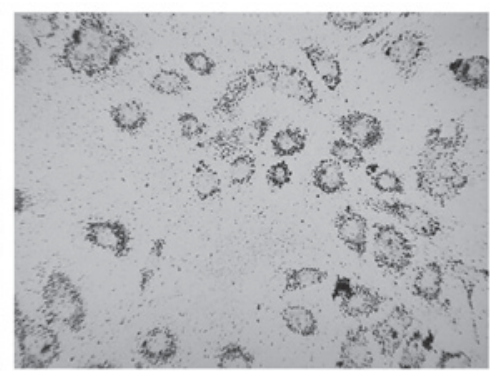

FFAs+lirag+compound C

Figure 10. Oil red O staining of FFA-exposed L-02 cells following treatment with liraglutide and compound C (magnification, x400). FFA, free fatty acid; lirag, liraglutide.
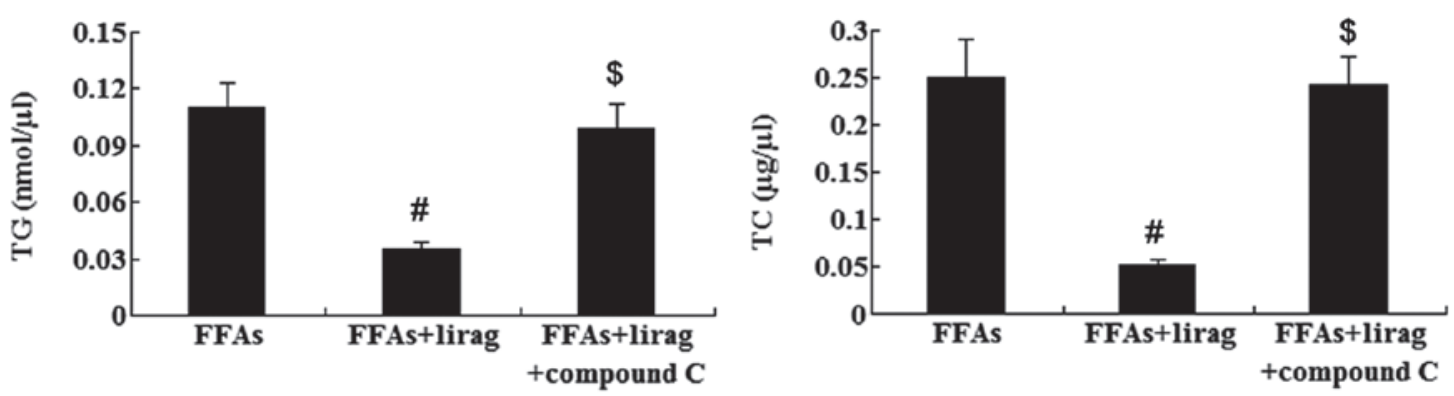

Figure 11. Intracellular levels of TG and TC in FFA-exposed L-02 cells following treatment with liraglutide and compound C. ${ }^{\#} \mathrm{P}<0.01 \mathrm{vs}$. FFA group; ${ }^{\circledR} \mathrm{P}<0.01$ vs. FFAs + lirag group. TG, triglyceride; TC, total cholesterol; FFA, free fatty acid; lirag, liraglutide.
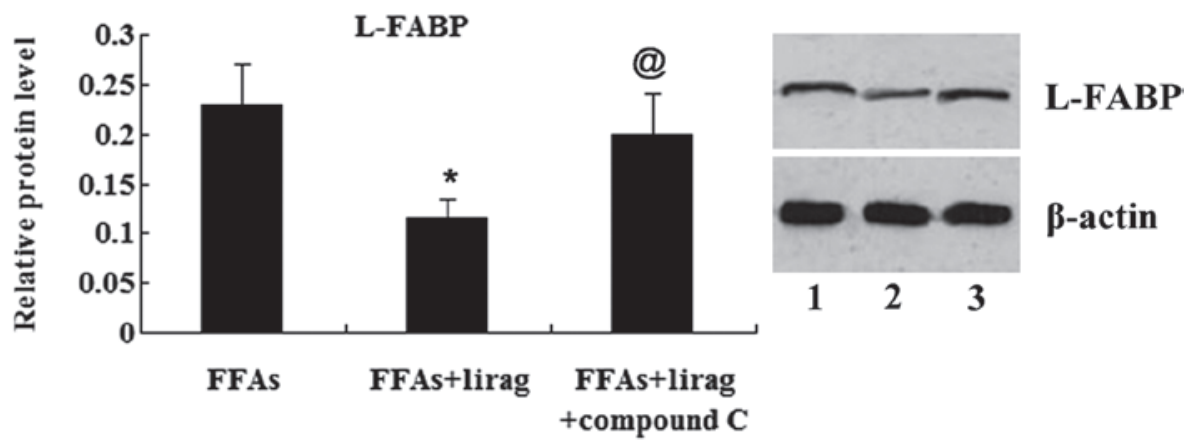

Figure 12. Expression of L-FABP protein in FFA-exposed L-02 cells following treatment with liraglutide and compound C. $\beta$-actin expression was used as an internal control. Lane 1, FFA group; lane 2, FFAs + lirag group; lane 3, FFAs + lirag + compound C group. ${ }^{*} \mathrm{P}<0.05$ vs. FFA group; ${ }^{\circledR} \mathrm{P}<0.05$ vs. FFAs + lirag group. L-FABP, liver-type fatty acid-binding protein; FFA, free fatty acid; lirag, liraglutide. 
FFA-exposed L-02 cells; however, this effect was attenuated by treatment with $20 \mu \mathrm{M}$ compound C (Fig. 10). Furthermore, it was found that, compared with the liraglutide group, treatment with $20 \mu \mathrm{M}$ compound $\mathrm{C}$ induced elevated levels of TG and TC in the FFA-exposed L-02 cells $(\mathrm{P}<0.01)$ (Fig. 11). The results from the western blot analysis revealed that the expression of L-FABP was suppressed by liraglutide; however, this effect was abolished by the treatment of the FFA-exposed L-02 cells with compound C (Fig. 12).

\section{Discussion}

To simulate the NAFLD condition in vitro, L-02 normal human hepatocyte-derived cells were treated with $0.5 \mathrm{mM}$ FFAs (PA and OA in a 1:2 molar ratio) for $24 \mathrm{~h}$. The effect of liraglutide on the development of NAFLD was then examined in vitro.

The results from liraglutide clinical trials have demonstrated the effect of liraglutide on reductions in TG, low-density lipoprotein cholesterol and FFAs (14-19). Consistent with the previous in vivo studies, oil red $\mathrm{O}$ staining in the present study demonstrated that liraglutide attenuated the significant increase in intracellular lipid accumulation. In addition, a significant decrease in the content of TG and TC was observed when the FFA-exposed L-02 cells were incubated with liraglutide, as indicated.

L-FABP plays a key role in the fatty acid metabolism of the liver. It has recently been reported that L-FABP constitutes a novel diagnostic marker for detecting NAFLD, and the serum and hepatic expression levels of L-FABP were significantly upregulated in NAFLD patients compared with those in control subjects $(20,21)$. In the present study, it was found that the increased expression of L-FABP in FFA-exposed L-02 cells could be suppressed by liraglutide. These findings together demonstrate that liraglutide has a potential function in improving NAFLD in vitro.

The AMPK/SREBP1 pathway plays an important role in the development of orotic acid-induced fatty liver (22). AMPK is a heterotrimeric enzyme complex that is involved in a variety of biological activities that normalize glucose, lipid and energy imbalances. SREBP1 is a transcription factor responsible for fatty acid synthesis (23). It has been demonstrated that AMPK phosphorylation can inhibit the expression of SREBP1 $(12,24,25)$, whereas the inhibition of AMPK augments the expression of SREBP1, thus leading to the activation of lipogenesis (26). In the present study, FFA-exposed L-02 cells were treated with various concentrations of liraglutide, and the results from the western blot analysis revealed that liraglutide affected the AMPK/SREBP1 pathway in a dose-dependent manner. The aim of the next part of the study was to examine whether the AMPK/SREBP1 pathway mediated the liraglutide-induced reduction in fatty degeneration.

The AMPK-mediated mechanism could be suppressed by compound C, an inhibitor of AMPK $(27,28)$. In the present study, it was found that compound $\mathrm{C}$ blocked the inhibitory effect of liraglutide on the increases in intracellular lipid accumulation, TG and TC content, as well as the elevated expression of L-FABP induced by FFAs. These results indicate that the effect of liraglutide on reducing fatty degeneration was mediated by the AMPK/SREBP1 pathway.
In conclusion, the present study has demonstrated that liraglutide can reduce the fatty degeneration induced by FFAs in hepatocytes. This effect may be partially mediated by the AMPK/SREBP1 pathway.

\section{References}

1. Clark JM and Diehl AM: Hepatic steatosis and type 2 diabetes mellitus. Curr Diab Rep 2: 210-215, 2002

2. Bedogni G, Miglioli L, Masutti F, Tiribelli C, Marchesini G and Bellentani S: Prevalence of and risk factors for nonalcoholic fatty liver disease: The Dionysos nutrition and liver study. Hepatology 42: 44-52, 2005.

3. Browning JD, Szczepaniak LS, Dobbins R, Nuremberg P, Horton JD, Cohen JC, Grundy SM and Hobbs HH: Prevalence of hepatic steatosis in an urban population in the United States: Impact of ethnicity. Hepatology 40: 1387-1395, 2004.

4. Vernon G, Baranova A and Younossi ZM: Systematic review: The epidemiology and natural history of non-alcoholic fatty liver disease and non-alcoholic steatohepatitis in adults. Aliment Pharmacol Ther 34: 274-285, 2011.

5. Fan JG: An introduction of strategies for the management of nonalcoholic fatty liver disease (NAFLD) recommended by Asia Pacific Working Party on NAFLD. Zhonghua Gan Zang Bing Za Zhi 15: 552-553, 2007 (In Chinese).

6. Petta S, Muratore C and Craxì A: Non-alcoholic fatty liver disease pathogenesis: The present and the future. Dig Liver Dis 41: 615-625, 2009.

7. Schwenger KJ and Allard JP: Clinical approaches to non-alcoholic fatty liver disease. World J Gastroenterol 20: 1712-1723, 2014.

8. Knudsen LB, Nielsen PF, Huusfeldt PO, Johansen NL, Madsen K, Pedersen FZ, Thøgersen H, Wilken M and Agers $\varnothing$ H: Potent derivatives of glucagon-like peptide-1 with pharmacokinetic properties suitable for once daily administration. J Med Chem 43: 1664-1669, 2000

9. Gao H, Xu L, Li D, Guang L and Deng W: Effects of glucagon-like peptide- 1 on liver oxidative stress, TNF- $\alpha$ and TGF- $\beta 1$ in rats with non-alcoholic fatty liver disease. Nan Fang Yi Ke Da Xue Xue Bao 33: 1661-1664, 2013 (In Chinese).

10. Olaywi M, Bhatia T, Anand S and Singhal S: Novel anti-diabetic agents in non-alcoholic fatty liver disease: A mini-review. Hepatobiliary Pancreat Dis Int 12: 584-588, 2013.

11. Morrison A, Yan X, Tong C and Li J: Acute rosiglitazone treatment is cardioprotective against ischemia-reperfusion injury by modulating AMPK, Akt, and JNK signaling in nondiabetic mice. Am J Physiol Heart Circ Physiol 301: H895-H902, 2011.

12. Zhou G, Myers R, Li Y, Chen Y, Shen X, Fenyk-Melody J, Wu M, Ventre J, Doebber T, Fujii N, et al: Role of AMP-activated protein kinase in mechanism of metformin action. J Clin Invest 108: 1167-1174, 2001.

13. Zhang L, Yang M, Ren H, Hu H, Boden G, Li L and Yang G: GLP-1 analogue prevents NAFLD in ApoE KO mice with diet and Acrp30 knockdown by inhibiting c-JNK. Liver Int 33: 794-804, 2013.

14. Buse JB, Rosenstock J, Sesti G, Schmidt WE, Montanya E, Brett JH, Zychma M and Blonde L; LEAD-6 Study Group: Liraglutide once a day versus exenatide twice a day for type 2 diabetes: A 26-week randomised, parallel-group, multinational, open-label trial (LEAD-6). Lancet 374: 39-47, 2009.

15. Marre M, Shaw J, Brändle M, Bebakar WM, Kamaruddin NA, Strand J,Zdravkovic M, Le Thi TD and Colagiuri S; LEAD-1 SU Study Group: Liraglutide, a once-daily human GLP-1 analogue, added to a sulphonylurea over 26 weeks produces greater improvements in glycaemic and weight control compared with adding rosiglitazone or placebo in subjects with Type 2 diabetes (LEAD-1 SU). Diabet Med 26: 268-278, 2009.

16. Nauck M, Frid A, Hermansen K, Shah NS, Tankova T, Mitha IH, Zdravkovic M, Düring M and Matthews DR; LEAD-2 Study Group: Efficacy and safety comparison of liraglutide, glimepiride, and placebo, all in combination with metformin, in type 2 diabetes: The LEAD (liraglutide effect and action in diabetes)-2 study. Diabetes Care 32: 84-90, 2009.

17. Garber A, Henry R, Ratner R, Garcia-Hernandez PA, Rodriguez-Pattzi H, Olvera-Alvarez I, Hale PM, Zdravkovic M and Bode B; LEAD-3 (Mono) Study Group: Liraglutide versus glimepiride monotherapy for type 2 diabetes (LEAD-3 Mono): A randomised, 52-week, phase III, double-blind, parallel-treatment trial. Lancet 373: 473-481, 2009. 
18. Zinman B, Gerich J, Buse JB, Lewin A, Schwartz S, Raskin P, Hale PM, Zdravkovic $M$ and Blonde L; LEAD-4 Study Investigators: Efficacy and safety of the human glucagon-like peptide-1 analog liraglutide in combination with metformin and thiazolidinedione in patients with type 2 diabetes (LEAD-4 Met+TZD). Diabetes Care 32: 1224-1230, 2009.

19. Russell-Jones D, Vaag A, Schmitz O, Sethi BK, Lalic N, Antic S, Zdravkovic M, Ravn GM and Simó R; Liraglutide Effect and Action in Diabetes 5(LEAD-5) met+SU Study Group: Liraglutide vs insulin glargine and placebo in combination with metformin and sulfonylurea therapy in type 2 diabetes mellitus (LEAD-5 met+SU): A randomised controlled trial. Diabetologia 52: 2046-2055, 2009.

20. Özenirler S, Degertekin CK, Erkan G, Elbeğ Ş, Tuncer C, Kandilc U and Akyol G: Serum liver fatty acid binding protein shows good correlation with liver histology in NASH. Hepatogastroenterology 60: 1095-1100, 2013.

21. Higuchi N, Kato M, Tanaka M, Miyazaki M, Takao S, Kohjima M, Kotoh K, Enjoji M, Nakamuta M and Takayanagi R: Effects of insulin resistance and hepatic lipid accumulation on hepatic mRNA expression levels of apoB, MTP and L-FABP in non-alcoholic fatty liver disease. Exp Ther Med 2: 1077-1081, 2011.

22. Jung EJ, Kwon SW, Jung BH, Oh SH and Lee BH: Role of the AMPK/SREBP-1 pathway in the development of orotic acid-induced fatty liver. J Lipid Res 52: 1617-1625, 2011.
23. Srivastava RA, Pinkosky SL, Filippov S, Hanselman JC, Cramer CT and Newton RS: AMP-activated protein kinase: An emerging drug target to regulate imbalances in lipid and carbohydrate metabolism to treat cardio-metabolic diseases. J Lipid Res 53: 2490-2514, 2012.

24. Porstmann T, Santos CR, Griffiths B, Cully M, Wu M, Leevers S, Griffiths JR, Chung YL and Schulze A: SREBP activity is regulated by mTORC1 and contributes to Akt-dependent cell growth. Cell Metab 8: 224-236, 2008.

25. Shklyaev S, Aslanidi G, Tennant M, Prima V, Kohlbrenner E, Kroutov V, Campbell-Thompson M, Crawford J, Shek EW, Scarpace PJ and Zolotukhin S: Sustained peripheral expression of transgene adiponectin offsets the development of diet-induced obesity in rats. Proc Natl Acad Sci USA 100: 14217-14222, 2003.

26. You M, Matsumoto M, Pacold CM, Cho WK and Crabb DW: The role of AMP-activated protein kinase in the action of ethanol in the liver. Gastroenterology 127: 1798-1808, 2004.

27. Gundewar S, Calvert JW, Jha S, Toedt-Pingel I, Ji SY, Nunez D, Ramachandran A, Anaya-Cisneros M, Tian R and Lefer DJ: Activation of AMP-activated protein kinase by metformin improves left ventricular function and survival in heart failure. Circ Res 104: 403-411, 2009.

28. Sasaki H, Asanuma H, Fujita M, Takahama H, Wakeno M, Ito S, Ogai A, Asakura M, Kim J, Minamino T, et al: Metformin prevents progression of heart failure in dogs: Role of AMP-activated protein kinase. Circulation 119: 2568-2577, 2009. 